\title{
The British Eclipse Expeditions of May 9, I929.
}

By Prof. F. J. M. Stratton.

BY the time these notes appear in print, the facts on May 9 will be known of the eleven expeditions from France, Germany, Great Britain, Holland, Japan, and the United States which are

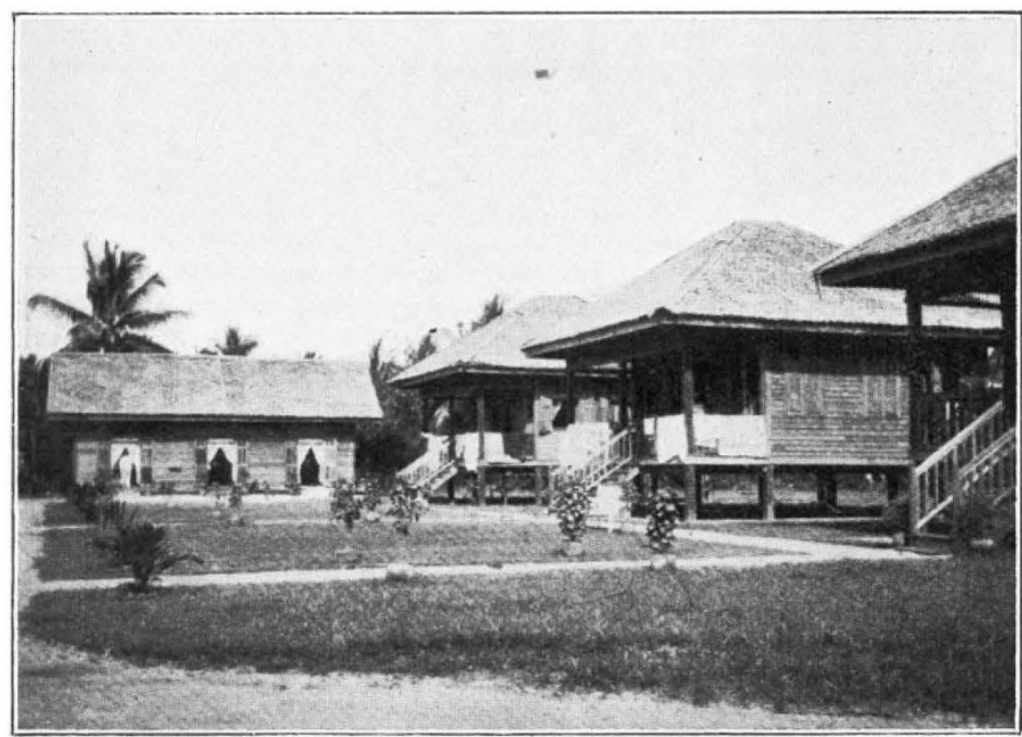

FIG. 1.-British observers' camp at Pattani.

at present scattered on the line Sumatra-KedahSiam-Cambodia-the Philippines. News can only be supplied here of the Japanese expedition under Prof. Sotome to Titra in Kedah, of the German expedition under Dr. Rosenberg to Khoke Bhodi in Siam, and of the two British expeditions to Alor Star in Kedah and to Pattani in Siam. In all cases it can be said that preparations are well in hand, attended so far by no serious delays or troublesome mischances.

Shelters of atap palm, in many cases supplemented by canvas or other linings to get the effect of a double roof, cover the instruments; preliminary adjustments are made and weather conditions do not seem likely unduly to hinder the final adjustments. So far as can be gathered by comparison with present weather conditions, prospects are most favourable at Pattani: their Majesties the King and Queen of Siam are to visit the British camp there for the eclipse, as in 1875 the then King of Siam observed the eclipse from Sir Arthur Schuster's camp.

In Kedah the Resident Adviser to the Regent has done everything to facilitate the work of the expeditions. Dr. and Mrs. Jackson are staying at the Residency and the eclipse camp is close by. The necessary electric current required for Dr. Carroll's comparison arc spectra, and for the mercury lamp to be used by Dr. Aston for the interferometer, has been obtained from the town mains, and through the kindness of the Regent all constructional work required has been done by the P.W.D. Photographic troubles have been largely met by the kind permission of the medical authorities to make use of the dark-room facilities at the local hospital.

In Siam the conditions are much more difficult, but the Siamese authorities have done everything possible to facilitate the work of the observers. At each place the Government has erected a hut camp with messroom, office, kitchen, six rooms and servants' quarters, lit by electric light. All constructional material required and labour have been supplied, arrangements made to guard the eclipse camps and to meet the requirements of the observers in the matter of electric current by the provision of portable electric plants. His Majesty the King of Siam appointed a special reception committee charged with the duty

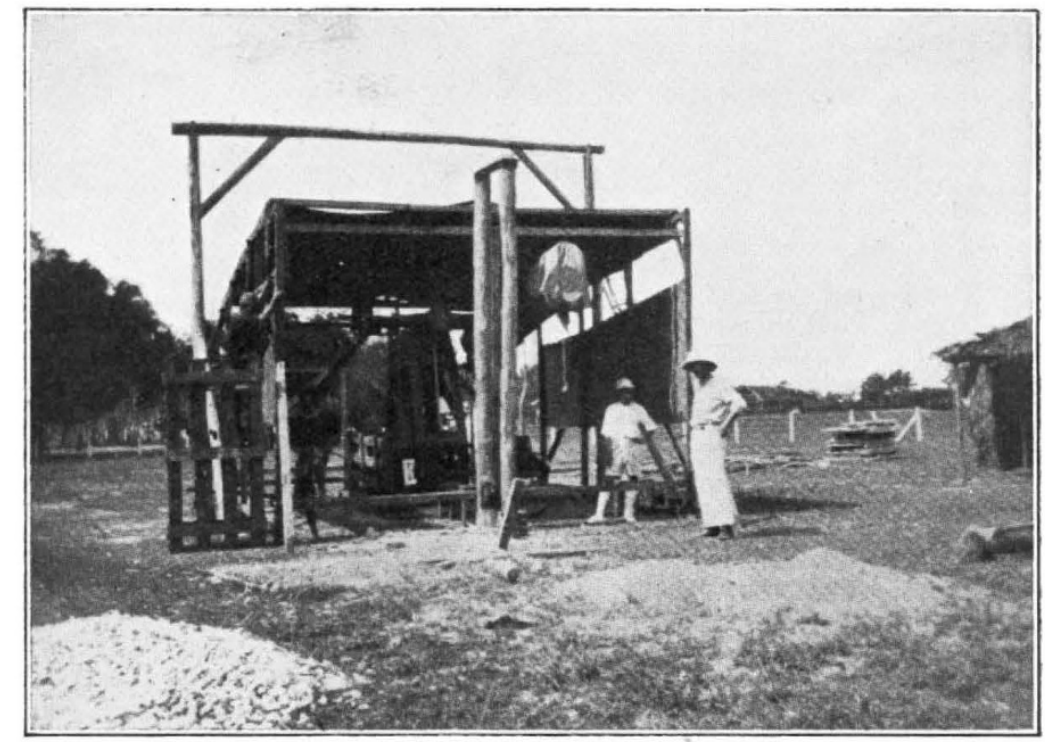

FIG. 2.-Telescope in position at Pattani.

of helping the astronomers in every way, and right well have they carried out their allotted task. All the local authorities in their turn have added to the obligations that the expeditions are under to the Siamese people. Photographic difficulties are not 
insuperable, though real, in this hot climate. A good deal can be done to meet them with ice from the local factory; and an efficient cooling plant, specially designed to meet the requirements of an eclipse dark room, should make matters still easier by providing a good supply of cooled water.

Most of the personnel for the actual observations have already arrived, but special mention must be made here of the services to the British expeditions of their honorary secretary, Col. J. Waley Cohen. Not only did he thoroughly explore both sides of the peninsula in 1928, bringing back valuable information as to meteorological conditions and as to local possibilities for eclipse preparations-and incidentally he interested many influential people in the coming eclipse-but he also arrived in the East this year ahead of the observers and made all the preparatory arrangements, so that a great deal was already done and in hand when they arrived. At Pattani, Col. Waley Cohen has also continued to relieve the scientists of the expedition of all worries about such matters as messing, local financial arrangements (not easy when there is no bank within many hours' journey of the camp), and the multitudinous details which have to be attended to, if matters are to go smoothly.

The accompanying photographs, taken by Dr. Royds, director of the Kodaikanal Observatory, show (Fig. 1) the special camp erected for the observers to live in, and (Fig. 2) the astrographic telescope from Greenwich in course of erection with Mr. P. J. Melotte's instruments, including a coronagraph of $19 \mathrm{ft}$. focal length with a direct vision prism for the first and second flash, three spectrographs, and a double tube camera with a Nicol prism in front of one object glass for a polariscopic study of the corona. The party of the observers and assistants on the day of the eclipse will be twelve. In addition to those above mentioned and myself, Prof. E. Barnes and W. F. Kibble, of Madras, have already been at the camp for some days and given valuable help.

\section{Einstein's and other Unitary Field Theories: An Explanation for the General Reader.} By Prof. H. T. H. Piaggio.

II.

\section{Geometry on a Sphere.}

$\mathrm{T}$ HE leading ideas of the geometry that Einstein chose (Riemannian) can be made clear by considering the properties of a geographical globe (Fig. 1) on which are marked the meridians and parallels of latitude. These divide the surface into what we may call curvilinear rectangles. But these rectangles are not all of the same size or shape. For consider two points with the same latitude but

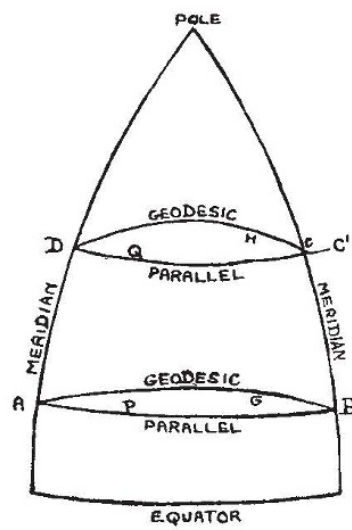

Frg. 1. with longitudes differing by one degree. The distance between them depends upon the latitude; it is greatest at the equator and zero at the poles. Thus APB is greater than DQC. For a sphere the distance between two points with the same longitude (i.e. on the same meridian), but with latitudes differing by one degree, is constant, but if our globe (like the earth itself) is flattened at the poles, this distance will again depend upon the latitude.

In either case, we cannot find the distance between two points $\mathrm{A}$ and $\mathrm{C}$ on the globe merely by knowing the differences of their latitudes and their longitudes, whereas in a plane the distance between two points is determined solely by a knowledge of the differences of their $x$ and of their $y$ co-ordinates. This is what is meant by the rather alarming statement that the sphere has a Riemannian metric, while the plane has a Euclidean one. (In mathematical symbols, $d s^{2}=d x^{2}+d y^{2}$ shows a Euclidean metric, but $d s^{2}=g_{11} d x^{2}+g_{22} d y^{2}$ shows a Riemannian metric, provided that $g_{11}$ and $g_{22}$ are functions of $x$ and $y$, or of either of them.)

It is not only a plane that has a Euclidean metric. Take a piece of squared paper, and roll it up, or bend it (without stretching or tearing) into as queer a shape as you please. The squares drawn on it remain all of the same size as before, hence the metric is still Euclidean. Such a surface is said to have zero Gaussian curvature, although it is what an ordinary person would call curved. The real distinction between it and a sphere is that the squared paper can be flattened out again, whereas it is impossible to flatten out a sphere or a piece of it (as may be easily verified with a piece of a broken rubber ball). Another way of putting this is to say that any attempt to make a flat map of the earth must be imperfect and give a distorted representation, as is obvious on Mercator's chart near the poles.

A well-known problem in geography or navigation is to determine the shortest route that can be traversed between two points on the earth's surface. On a model globe we can determine this experimentally by stretching a piece of string between these two points. It will be found that it will lie in what is called a Great Circle, namely, one the plane of which passes through the centre of the earth. It is important to notice that it is not the same as a parallel of latitude. In fact a ship that has to sail between two points $\mathrm{A}$ and $\mathrm{B}$ on the same parallel APB (north of the equator) will, to follow the Great Circle AGB, have to sail north of this parallel and then come back to it, a method rather tiresome to navigators, as it entails a continual change of direction (as measured by compass bearing). A Great Circle on a sphere has one of the properties of a straight line in a plane, namely, that of being a geodesic or shortest distance between

No. 3110, VoL. 123] 